\title{
Reading different orthographies: an fMRI study of phrase reading in Hindi-English bilinguals
}

\author{
Uttam Kumar · Tanusree Das · Raju S. Bapi · \\ Prakash Padakannaya $\cdot$ R. Malatesha Joshi • \\ Nandini C. Singh
}

Published online: 8 April 2009

(C) Springer Science+Business Media B.V. 2009

\begin{abstract}
The aim of the present study was to use functional imaging to compare cortical activations involved in reading Hindi and English that differ markedly in terms of their orthographies by a group of late bilinguals, more fluent in Hindi (L1) than English (L2). English is alphabetic and linear, in that vowels and consonants are arranged sequentially. In contrast, Hindi, written in Devanagari, is an alphasyllabary and non-linear writing system wherein vowels are placed around consonants making it a visually complex script. Additionally, the grapheme to phoneme mapping in English is opaque while Devanagari is transparent. Effects of reading fluency were seen in significantly slower reading times and direct EnglishHindi comparison showed left putamen activation for the less fluent language (English). Direct Hindi-English orthography comparisons revealed activation in the temporal pole and caudate nucleus of the right hemisphere, cortical areas known to be involved in semantic and visual processing. We also find activation in right superior temporal gyrus, which we attribute to the syllabic rhythm of Hindi. Our
\end{abstract}

Uttam Kumar and Tanusree Das are joint first authors.

U. Kumar · T. Das · N. C. Singh $(\bowtie)$

National Brain Research Centre, NH-8, Nainwal Mode, Manesar 122 050, India e-mail: nandini@nbrc.ac.in

R. S. Bapi

Department of Computer and Information Sciences, University of Hyderabad, Hyderabad 500 046, India

P. Padakannaya

Department of Psychology, University of Mysore, Mysore 570 006, India

R. M. Joshi

College of Education in Human Development, Texas A \& M University, College Station, TX 77843, USA 
results suggest increased visuo-spatial demands for processing Hindi as observed in other visually complex orthographies.

Keywords Bilinguals · Devanagari · English · fMRI · Hindi · Orthography ·

Reading

\section{Introduction}

A remarkable accomplishment of many bilinguals is the acquisition of reading skills in distinct scripts. Unlike French-English or Italian-English bilinguals, wherein the Roman script is used for both languages, Chinese-English and Hindi-English bilingual readers learn to read two distinct orthographies. Research attempts to understand the mechanisms underlying reading in such bilinguals have been motivated by two hypotheses, namely the universal language hypothesis (Clarke, 1980; Goodman, 1973; Perfetti, Zhang, \& Berent, 1992) and the language-specific hypothesis (Fiez, 2000; Gottardo, Yan, Siegel, \& Wade, 2001; Holm \& Dodd, 1996; Koda, 1994; Neville et al., 1998; Vaid, 2002; Vaid \& Hull, 2002; Wang \& Geva, 2003; Weinreich, 1953). Since all writing systems represent spoken language, there are universal principles governing the written representation of language (Perfetti, 2003). The universal language system theory therefore suggests a common cognitive and neuro-anatomical network for reading across languages. On the other hand, the visual forms that represent units of spoken language vary across writing systems. The language-specific hypothesis therefore suggests separate processing networks for different orthographies (Meschyan \& Hernandez, 2006; Tan et al., 2003).

The advent of functional neuroimaging as a non-invasive high-resolution anatomical technique has provided researchers of language and neuroscience a unique opportunity to study and validate such hypotheses. On one hand neuroimaging studies have shown that there are principles governing the written representation of language that are universal (Perfetti et al., 1992) and that degree of proficiency or fluency in a language is a critical factor over and above the visuo-spatial demands or transparency of the orthography with less proficient language producing more left prefrontal and parietal activation (Chee, Hon, Lee, \& Soon, 2001).

On the other hand, there is also sufficient evidence suggesting that differences in visual forms play a role in cortical activation. For example, a comparison of languages like English and French that use the alphabetic writing system with Chinese, Japanese Kana and Kanji by Bolger, Perfetti and Schneider (2005) have suggested that writing systems engage the same areas in terms of gross cortical regions, but activation within those regions differs across writing systems. Their analysis of over 150 studies on visual word recognition revealed regions associated with orthography, phonology and semantics of a writing system. Orthography associated activations included bilateral occipital/posterior fusiform gyrus (BA 18, 37/19), left mid-fusiform and posterior inferior temporal gyrus (BA 37). Areas associated with phonological processing included superior temporal sulcus/inferior parietal lobe (BA 22/40/39), inferior frontal sulcus/insula/premotor cortex (BA 45/ 6/9). Activations related to semantic processing included anterior fusiform/inferior 
temporal gyrus, middle temporal gyrus, anterior inferior frontal gyrus (BA 37/21, 44 ). In the case of bilinguals, most of these studies have focused on languages that are both written alphabetically [English-Spanish (Meschyan \& Hernandez, 2006), English-French (Frenck-Mestre, Anton, Roth, Vaid, \& Viallet, 2005; Kim, Relkin, Lee, \& Hirsch, 1997), English-Italian (Perani et al., 1998)] and suggest similar patterns of cortical activation (Frenck-Mestre et al., 2005). However studies on bilinguals reading different scripts have shown that the earlier learnt language/ orthography affects reading in a later learned language (Liu, Dunlap, Fiez, \& Perfetti, 2007; Perfetti, Liu, Fiez, Nelson, \& Bolger, 2007; Tan et al., 2003). These results have primarily emerged from studies with Chinese-English bilinguals and have clearly shown differences in cortical activation specific to reading different scripts.

In addition, these studies have also shown that cortical activation patterns observed are influenced by visual form. As a result, the second language activation patterns seen in bilinguals differ from those seen in monolingual readers of the same language (Tan et al., 2003). For instance, Perfetti et al. (2007) studied fluent Chinese-English bilinguals who were native Chinese speakers fluent in English and found that a morpho-syllabic language like Chinese elicited distinct neural correlates namely the left middle frontal gyrus (LMFG) during reading. A Chinese logographic character has a square shape that represents a syllable. This structure is spatially highly complex and hence requires higher order spatial processing, which involves left middle frontal gyrus not seen in English word reading by monolinguals. However, the Chinese-English bilinguals also showed LMFG activation while reading in English. Thus, they concluded that neural systems of reading in English (the second language) was shaped by native language reading. They also suggested that the overlapping reading network in these two languages was observed because the bilinguals were highly proficient in both languages. However, in a second study on English-Chinese bilinguals (Nelson, Liu, Fiez, \& Perfetti, 2005), the activation pattern for reading in Chinese by native English readers, again showed bilateral MFG which led them to believe that an alphabetic script (English) was not adept to assimilate a more complex morpho-syllabic script like Chinese.

The Chinese-English studies thereby established that for a given task, the orthography and visual form of the writing system influenced cortical activation. Tan and colleagues (Tan et al., 2005), in a meta-analysis of phonological processing of Chinese characters found high concordance of activation across nineteen studies in left middle frontal gyrus (BA 9). Other activations were observed in bilateral occipital gyri (BA 18), bilateral fusiform gyrus (BA 37), left medial frontal gyrus (BA 6, 9), left inferior frontal gyrus (BA 47) and dorsal aspect of left inferior parietal cortex (IPL, BA 40). For phonological processing of alphabetic words, highest convergence was obtained in left inferior frontal gyrus (BA 44) and other activations were obtained in left fusiform gyrus (BA 37), cerebellum, left midsuperior temporal regions (BA 21, 42), ventral aspect of left inferior parietal cortex (involving supramarginal gyrus, BA 40), left medial frontal gyrus (BA 6, 8), right superior temporal gyrus (BA 22) and right mid-inferior occipital gyrus (BA 18).

The dorsal aspect of left IPL was suggested to be a temporary store for phonological information in working memory in Chinese (Ravizza, Delgado, Chein, 
Becker, \& Fiez, 2004). In alphabetic words however, higher activations in left IFG, left temporoparietal region (Booth et al., 2004) and supplementary motor area (SMA, BA 6) correspond to grapheme-to-phoneme conversion (Fiez, Balota, Raichle, \& Petersen, 1999) and subvocal rehearsal component of phonological processes (Smith \& Jonides, 1999). The left medial fusiform gyrus activation is more relevant to Chinese whereas the left lateral fusiform gyrus is associated with English (Dietz, Jones, Gareau, Zeffiro, \& Eden, 2005). Hence, overlapping cortical areas like left temporo-occipital circuits that mediate phonological processing are present in both alphabetic and logographic scripts. It is the surface form or the visuo-spatial complexity of different orthographies that lead to characteristic activations.

The role of orthographic transparency and language proficiency in reading different writing systems was examined by Meschyan and Hernandez (2006) in Spanish-English bilinguals. They compared cortical activations while reading Spanish and English by Spanish-English bilinguals, who were more proficient in English, their second language. They hypothesized that there should be more activity recorded from regions involved in articulatory motor processing (SMA/ cingulate, putamen, insula) while participants read in a less proficient orthography and did find such results for reading in Spanish (which was the less proficient language of the participants) in their study. In addition, for Spanish which has a transparent orthography, they reported greater activity in left superior temporal gyrus (BA 22) a region implicated in assembled phonological strategy of reading (Paulesu et al., 2000), while for English, which has an opaque orthography, they reported greater activity in visual processing and word recoding regions such as left occipito-parietal border and right inferior parietal lobe (BA 40). Their behavioral results also showed that participants were significantly slower in reading words in the less proficient language, Spanish.

The purpose of our study is to build on the work described by Meschyan and Hernandez (2006), nevertheless, it differs from it in one major respect. In their study the effects of low reading fluency and orthographic transparency were confounded. The participants in their study were less proficient in Spanish, which also has a transparent orthography. They found high activation in speech-motor regions while reading Spanish, which could be either due to transparency or poor reading fluency or both. Having Hindi-English biliterate participants, more fluent in reading Hindi (transparent orthography) than English (opaque orthography) would allow us to juxtapose the relative effects of fluency versus orthographic transparency.

Hindi uses Devanagari, an ancient writing system widely used in South Asia. According to the 2001 census survey of India, out of the one billion Indian population, 33\% speak Hindi (Singh, Solanki, \& Bhatnagar, 2008), which is approximately 330 million. An economic survey conducted by the Government of India in 2004 (UNDP report, 2004) reported a national literacy rate of $61 \%$ suggesting that about 200 million use the Devanagari script. The Devanagari script, like almost all Indic writing systems, is derived from Brahmi, an abugida orthography (MacKenzie \& Tanaka-Ishii, 2007). An abugida is a segmental writing system wherein vowel notations are obligatorily associated with and built into the consonants. The Devanagari script has syllabic as well as alphabetic properties and 
hence called an alphasyllabary. The Devanagari script differs from the Roman derived English script in terms of grain size, transparency as well as orthographic layout (Padakannaya \& Mohanty, 2004; Patel, 2004; Padakannaya \& Joshi, 1995; Sproat \& Padakannaya, 2008; Vaid \& Gupta, 2002). The basic written unit is called akshara that stands for orthographic syllable: consonant(s) with inherent vowel /a/ or consonant(s) with vowel diacritics or simply vowel in its full form. Though akshara stands for a syllable, it can be visually analyzed into constituent phonemes thus making it different from a syllabary such as Kana. English letters are designed with basic geometric shapes like vertical, horizontal, diagonal lines, circles and use several symmetrical shapes. Devanagari characters, on the other hand, have a complex spatial organization and are asymmetric, free flowing and have highly intricate shapes. While English has a linear organization in that vowels and consonants are only written in a linear left-to-right fashion, in Devanagari, the consonants are mostly written in a linear left-to-right order (कमल, 'kamal', meaning lotus) and vowel signs are positioned nonlinearly above (खेल, 'khel', meaning to play), below (खुल, 'khul', meaning 'to open') or to either side of the consonants (खाल, 'khal', meaning 'skin'). In addition, for certain words in Devanagari, the vowel precedes the consonant in writing but follows it in speech (खिल, 'khil', meaning 'to blossom'). These qualities make reading in the Devanagari script rather complex and challenging. However, it is orthographically transparent with nearly perfect one to one correspondence between akshara and sound as opposed to English, which is irregular. In the present study we compared cortical activation patterns involved in phrase reading in Hindi and English by late bilinguals more proficient in reading Hindi. This is the first such fMRI study on Hindi-English bilinguals wherein we expected to find effects of (a) orthographic layout or visuospatial configuration of written form (b) orthographic transparency and (c) reading fluency. The specific objectives of the present study include the following: (1) To record common cortical activation patterns in reading Devanagari and English, which may indicate a universal neuronal network involved in reading, independent of variations in specific orthographies. (2) To juxtapose the results of the present study with that of Meschyan and Hernandez (2006) and interpolate the results in order to arrive at more valid conclusions on the relative influence of orthographic transparency and language fluency of biliterates on reading. (3) To look for the specific cortical activations that could be related to language/script-specific features of Hindi/Devanagari.

\section{Method}

\section{Participants}

Twelve right-handed Hindi (L1)-English (L2) late bilinguals (7M, 5F, mean age 28.4 years, SD 3.2 years, age range, 25-32 years) participated in this study. Handedness was assessed by the Edinburgh Inventory (Oldfield, 1971). Participants were neurologically intact with no history of neurological disorders. Participant characteristics and language background were assessed via a questionnaire, which 
included questions like, 'age', 'gender', 'age of native language acquisition', 'age and exposure to second language' and 'place of residence for longest period'. All the participants possessed at least a graduate degree and reported Hindi as their native language. Age of first exposure to English was at school at 8-9 years of age. They have been reading English since then and Hindi since 4 years of age. Currently, they read Hindi for 7-10 h a day and English for 0-2 h a day on an average. They also rated themselves lower in English reading than Hindi reading. Participants did not know any additional language other than Hindi and English. Informed consent was obtained from all participants in accordance with the guidelines of the human ethics committee of the National Brain Research Centre.

\section{Behavioral design}

To assess reading skills in English and Hindi, participants were asked to read two short passages aloud - the standard IPA (International Phonetic Alphabet) text for segmental coverage "The Northwind and the Sun" and its Hindi translation. Each passage consisted of eight sentences (114 words), which appeared in its entirety on the computer screen. Participants read the entire passage aloud. Reading time was measured by requiring a button press upon beginning to read and again upon conclusion and was used as a behavioral measure of reading fluency. This method has been used by Haller, Radue, Erb, Grodd and Kircher (2005). Error analysis of the reading data was based on errors marked by early Hindi-English bilinguals.

\section{fMRI design}

Most studies on reading have used word level tasks. While word-level tasks have been successful in providing insights into organization and processing of languages, they are constrained in that such paradigms are unable to provide insights into the different strategies that might be deployed during natural language processing (Poeppel, 1996). In keeping with the behavioral paradigm, for the present study, the ideal choice would have been overt reading of the same passages or sentences in the fMRI scanner. However overt reading for extended periods could give rise to correlated head movement, which is often a serious cause for concern in functional imaging data (Gracco, Tremblay, \& Pike, 2005). In order to minimize head movement we decided to use phrases, which were short enough to minimize head motion, but at the same time complex enough to be similar to sentence processing thereby invoking different aspects of language such as phonology, syntax, semantics, verbal short-term memory as well as basic visual recognition. Additionally, to avoid auditory feedback of subject's own voice (Callan et al., 2006), covert production, i.e., silent movement of articulators was used.

A block design was used to determine brain activity associated with covert reading of phrases in English and Hindi. Two runs of 16 blocks each were administered. Each run had four blocks of English and four of Hindi that alternated with those of rest. Each block lasted $20 \mathrm{~s}$ in which four phrases (three-word long) were presented at the rate of 1-phrase/5 s. Phrases were taken from a passage 'North wind and sun' and its Hindi translation and were matched for length and meaning across languages. During 


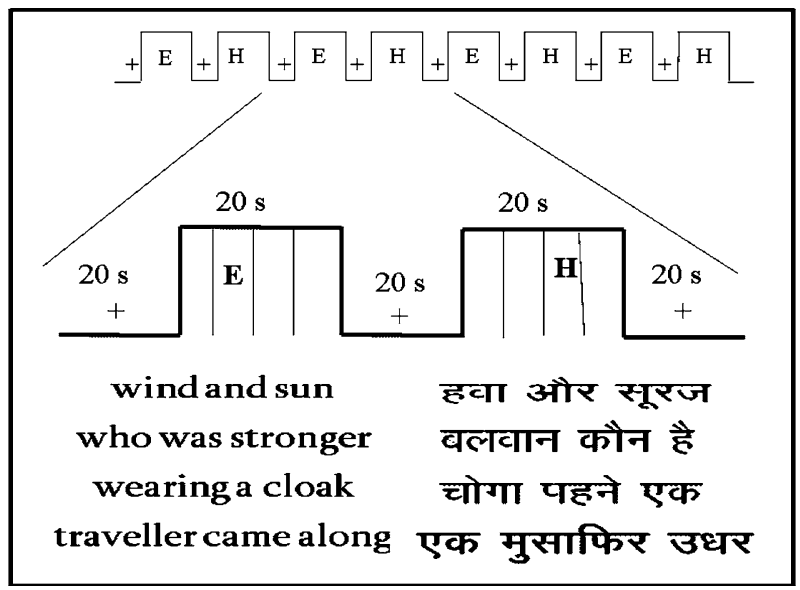

Fig. 1 Schematic showing block paradigm for fMRI task. Top panel an experimental session (run) comprised of 16 blocks wherein eight 'rest' blocks alternated with four 'English' and four 'Hindi' blocks. Middle panel each block was $20 \mathrm{~s}$ long. During 'rest' block, participants fixated on a fixation cross. During stimulus block, a three-word phrase was presented every $5 \mathrm{~s}$. All four phrases presented in a block were either in English or Hindi (extracted from 'North wind and sun' or its Hindi translation). Bottom panel representative phrases used in the English and Hindi blocks have been shown

rest blocks, fixation was maintained at a crosshair in the center of screen. Thus four phrases were shown in each block and the sequence and order of presentation of the phrase stimuli were randomly shuffled among the subjects. The semantic content of the phrases in both Hindi and English were kept the same. Each participant completed two such runs. During the rest condition, which was used as a baseline control condition, participants were instructed to look at a fixation-cross (Fig. 1). A total of 320 volumes were acquired from each participant.

Image acquisition

Scanning was conducted on a 3 Tesla Phillips MRI scanner equipped with echo planar imaging (EPI) and a standard head coil for radio frequency transmission and signal reception. Stimuli were retro-projected onto a screen outside the scanner and a head-mounted apparatus in the scanner. The presentation of written words was controlled by E-prime software running on an IBM-compatible computer located outside the scanner. Participants lay supine with their heads restrained by a soft strap. They were instructed to read silently, to move their articulators without any sound. High-resolution structural T1-weighted images covering the whole brain were acquired from all participants for anatomical localization. Functional images were acquired using a T2-weighted echo-planar sequence at 30 axial slices parallel to the AC-PC plane. (TR/TE $=2 \mathrm{~s} / 35 \mathrm{~ms}$, flip angle 90 degrees, FOV was $230 \mathrm{~mm}$ with $64 \times 64$ image matrix, yielding an in plane resolution of $3.59 \times 3.59 \mathrm{~mm}$. Slice thickness was $4 \mathrm{~mm}$ with $1 \mathrm{~mm}$ gap). A total of 320 volumes were acquired for each participant, which is in conjunction with other studies on reading (Meschyan \& Hernandez, 2006). 
fMRI analysis

The imaging data were analyzed using statistical parametric mapping SPM5 at Wellcome Department of Cognitive Neurology, London. The functional images were reoriented to set the origin near the intersection of the coronal plane through $\mathrm{AC}$ and the AC-PC line and then motion correction was performed with respect to the first functional image in each session. Anatomical image for each participant was co-registered with the first functional image and then normalized to the T1 template from the International Consortium for Brain Mapping (ICBM) Project. The resulting parameters were used for normalizing (Friston, Frith, Frackowiak, \& Turner, 1995) all the functional images into Talairach stereotaxic space (Talairach \& Tournoux, 1988). Spatial smoothing with a Gaussian kernel of $8 \mathrm{~mm}$ FWHM and temporal filtering (Gaussian low pass filter with $4 \mathrm{~mm}$ full-width at half maximum) was applied to the normalized images. The preprocessed data were analyzed using the general linear model framework (Friston et al., 1995). For each participant, the experimental settings (language task versus fixation) were modeled using boxcar functions convolved with the canonical hemodynamic response function. The canonical haemodynamic response function (HRF) is assumed to be the response of the system (as reflected by the MR signal) to a brief, intense period of neural stimulation. The HRF used in the SPM5 exhibits a rise that peaks around $6 \mathrm{~s}$, followed by an undershoot that persists for a considerable period (typically about $30 \mathrm{~s}$ ). In addition, six regressors (right, forward, up, pitch, roll and yaw) related to movement correction were used in the statistical model to account for any spurious activity related to head movements. The resulting $t$-maps [SPM (t)] from each participant were taken into a second-level analysis.

Group analysis was performed using the random effects approach (Penny, Holmes, \& Friston, 2003) as implemented in the SPM5 software. Contrast images computed from the subject-specific models were entered into a one-sample $t$-test at the second level. The voxel coordinates reported in Table 1 are MNI co-ordinates. Locations of peak activations along with their spatial extent are reported in Table 1 for various regions. Statistical thresholding at a significance level of $p=0.001$ (uncorrected) was applied for determining significant activations.

\section{Results}

\section{Behavioral}

The behavioral task was administered after the fMRI scan. As seen in Fig. 3a, the mean reading time for English $(50.8 \mathrm{~s}, \mathrm{SD}=9.793 \mathrm{~s})$ was significantly more than Hindi $(42.5 \mathrm{~s}, \mathrm{SD}=5.407 \mathrm{~s}$, paired $t$-test $p=0.04)$ suggesting that the participants were more fluent in reading Hindi (L1) as compared to English (L2). Contrastive error analysis which included word substitutions (for example, 'successed' instead of 'succeeded') and faulty pronunciations ('clock' instead of 'cloak') in the two languages, showed significantly greater $(p=0.0059)$ errors in reading English than 


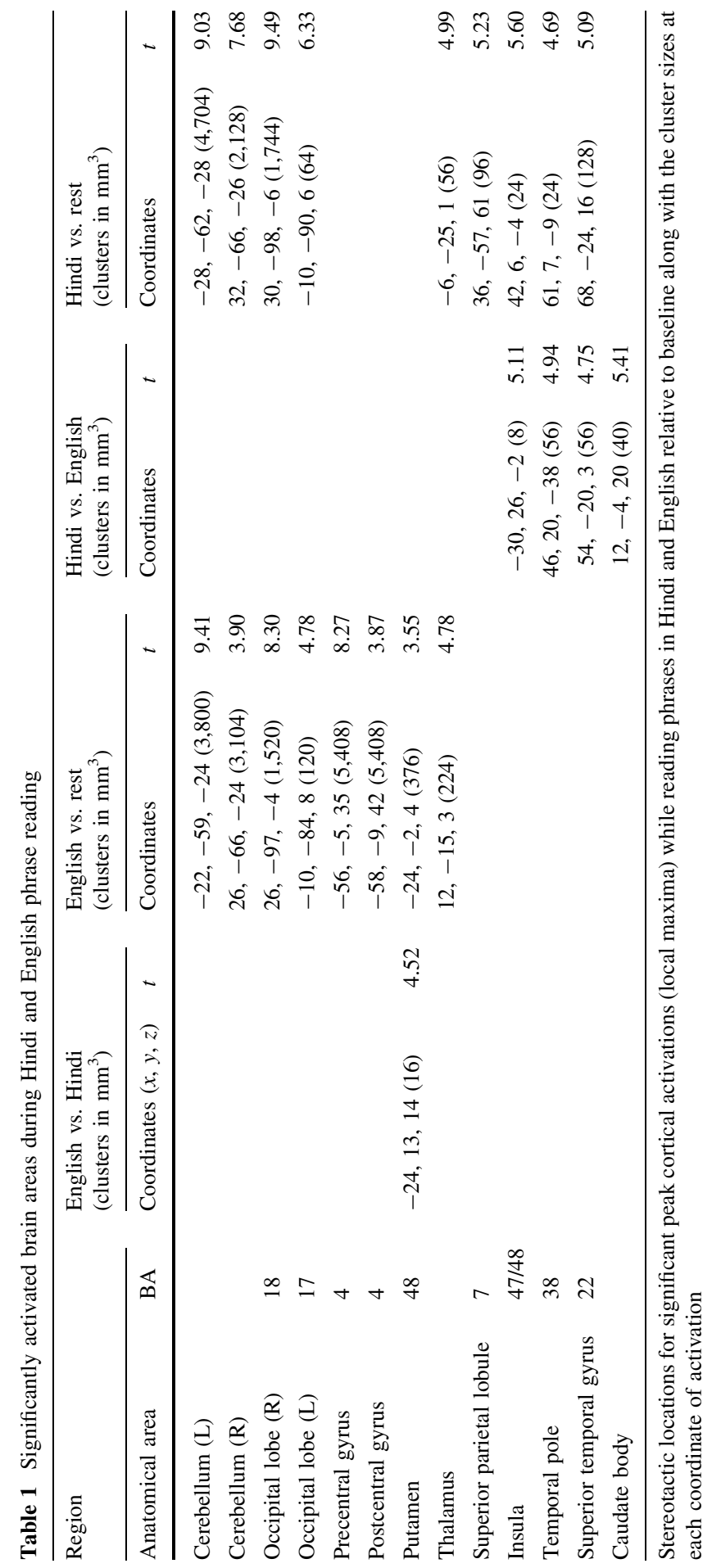


reading Hindi. Reading in English showed $4.3 \%$ errors while reading in Hindi showed $0.22 \%$ errors.

fMRI

Figure 2 displays the hemodynamic response obtained from the task epochs as indexed to the fixation baseline. Fig. 3a shows fMRI and behavioral results for fluency while Fig. $3 b$ shows effects of orthography. The colored areas comprise significantly activated voxels averaged across participants and measurement periods, as compared to the baseline condition.

As seen from Fig. 2 and Table 1, covert reading in both Hindi and English compared with baseline, led to common activation in bilateral cerebellum, bilateral occipital areas and thalamus. Distinct areas observed during the covert reading task in English were in left postcentral (BA 3) and precentral gyrus (BA 4) and left putamen. Additionally, for reading in Hindi activation was found in superior parietal lobule, insula, middle temporal gyrus (MTG)/superior temporal gyrus (STG) and temporal pole in the right hemisphere.

A comparison between English phrase reading relative to Hindi phrase reading revealed greater activation in left putamen. Hindi relative to English reading resulted in higher activation in right STG (BA 22), right temporal pole (BA 38), right caudate body and left insula (BA 47/48, Table 1).
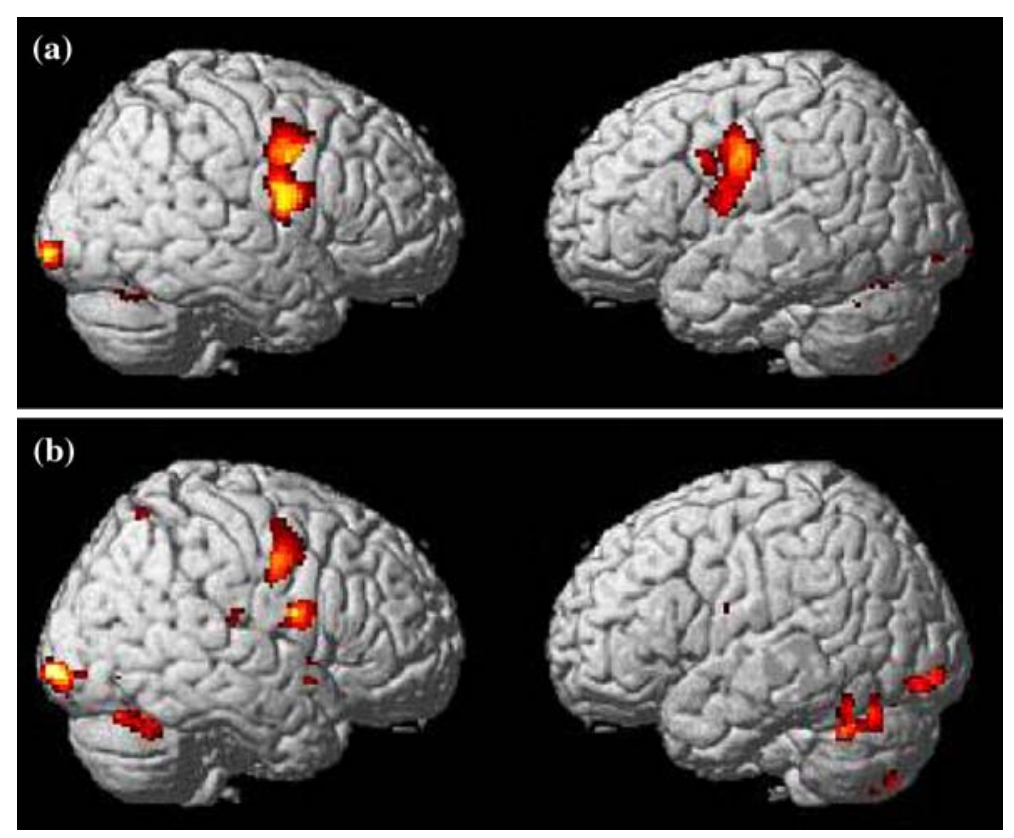

Fig. 2 Group activation maps for phrase reading. a English versus baseline. b Hindi versus baseline 
(a)

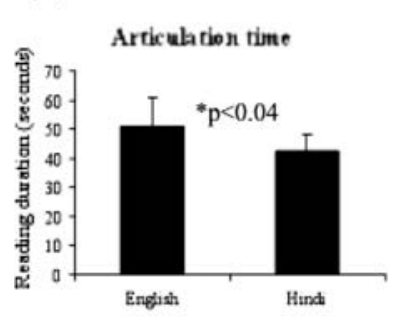

(b)

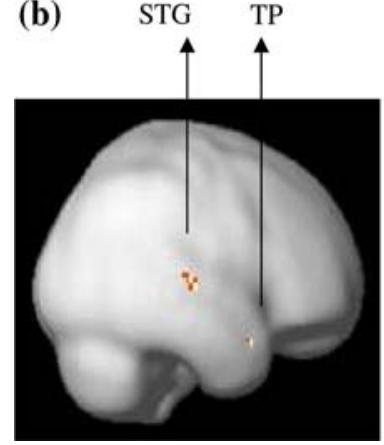

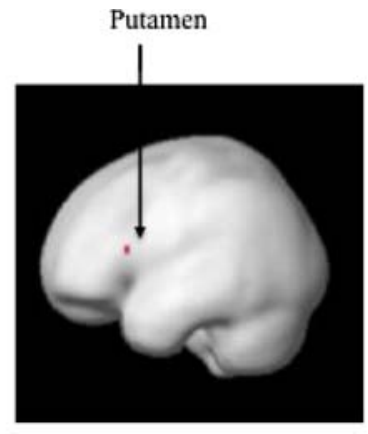

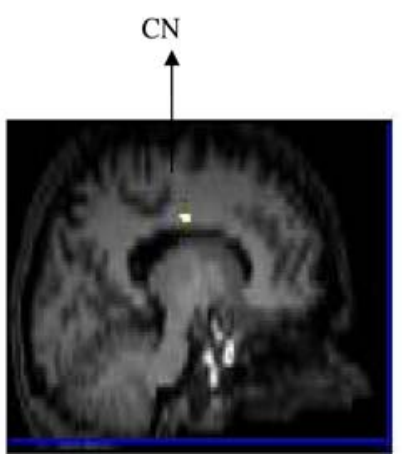

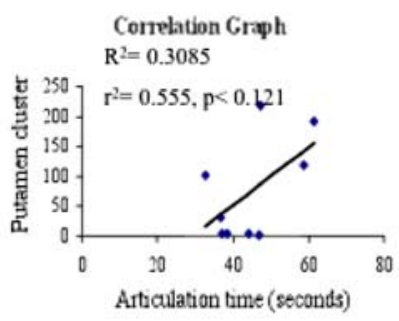

Insula

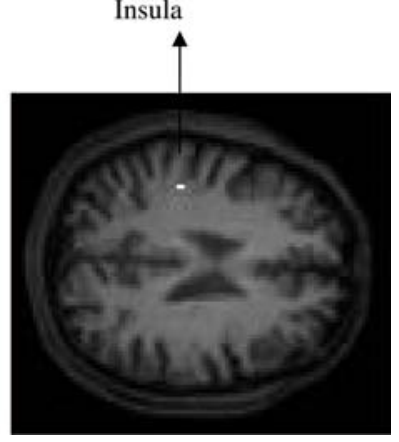

Fig. 3 a Effect of fluency. Effect of fluency was observed in left putamen in English $>$ Hindi comparison. Left panel reading times in Hindi-English late bilinguals were significantly higher in English than Hindi $(p=0.04)$. Middle panel rendering showing putamen activation (projected on surface) in English > Hindi. Right panel Pearson's correlation coefficient $\left(r^{2}\right)$ of reading times in English with extent of putamen activation ( $t$-value) was found to be $0.55(p=0.12) . R^{2}$ depicts correlation of data points with the fit. b Effects of orthography. Effects of orthography were seen in Hindi $>$ English comparisons. Increased activations in right superior temporal gyrus (STG), right temporal pole (TP), right caudate nucleus $(\mathrm{CN})$ and left insula were seen while reading Hindi

\section{Discussion}

The objective of this study was to examine the effects of (a) visuo-spatial layout (b) orthographic transparency and (c) fluency on neural activation patterns during covert reading of phrases in two writing systems, namely Hindi and English, wherein the orthographies differ markedly in visuo-spatial layout and transparency. The late bilinguals we chose differed significantly in their reading proficiencies in these two languages. Our results suggest an influence of all three factors on cortical activation patterns.

A comparison of neuroimaging studies on word reading has shown that there are principles governing the written representation of language that are universal (Perfetti et al., 1992). On the other hand, phrase and sentence processing are complex processes, capable of invoking different aspects of language such as phonology, syntax, semantics, verbal short-term memory as well as basic visual recognition. The bilateral occipital, motor and cerebellar activations observed in all the 12 participants during phrase reading in both English and Hindi are similar to 
the bilateral activation patterns reported for reading sentences in English and French (Bavelier et al., 1997; Frenck-Mestre et al., 2005) and suggest a universal reading network for phrase and sentence processing.

\section{Effects of fluency}

In keeping with earlier findings (Klein, Zatorre, Milner, Meyer, \& Evans, 1995), our results suggest the contribution of additional brain areas (left putamen) for articulating in the less fluent language. As shown in Fig. 3a the Hindi-English late bilinguals showed slower reading times for English. In addition, 10 out of the 12 subjects also showed activation in the left putamen. The Pearson's correlation co-efficient of reading times in English with the extent of activation ( $t$-value) in the putamen was found to be 0.55 ( $p<0.12$, Fig. 3a). Together, the behavioral and fMRI analysis suggests that the less practiced and therefore less fluent language results in slower reading times and left putamen activation, though it may be noted that putamen activation is based on 10 out of 12 subjects, which possibly accounts for the low $p$-value. This effect seems to be independent of whether the orthography in question is transparent or opaque. It will be interesting to see whether similar activation is observed in case of languages with non-phonetic scripts.

\section{Effects of visual form and orthographic transparency}

As described earlier and also seen in the example stimuli of Fig. 1, when compared to English, the spatial script of Devanagari consists of complex visual units, wherein vowels are arranged non-linearly around consonants. A reading task in Devanagari would therefore place increased processing load on the visuo-spatial processing system. One of the questions that naturally crops up in case of Indian alphasyllabaries is the interaction between visuo-spatial complexity and orthographic transparency. The visuo-spatial complexity of akshara (alphasyllabograph) does undermine the advantages of orthographic transparency in reading/writing and segmentation tasks in terms of both accuracy and speed. The scope of such a play off, however, is influenced by the nature of the specific segmentation task and language proficiency of individuals (Padakannaya, Rekha, Nigam, \& Karanth, 1993; Sproat \& Padakannaya, 2008; Vaid \& Gupta, 2002). In reading Tamil as well as Devanagari, processing of CV akshara with compound vowel ligature resulted in higher response times than for those with single vowel ligature (Sproat \& Padakannaya, 2008; Vaid \& Gupta, 2002). In terms of the reading accuracy, children commit more mistakes in reading akshara with vowel ligatures or consonant blends. Even while performing segmentation tasks, studies have reported that visuo-spatially complex word stimuli posed more difficulty, particularly in phoneme deletion and phoneme reversal tasks (Sheela, 2007). This interaction between transparency and visuo-spatial complexity of Devanagari has resulted in some interesting cortical activations.

A direct comparison of the orthographies of Hindi and English revealed activation of the temporal pole (BA 38, 7/12 subjects) and the caudate nucleus in the right hemisphere (6/12 subjects). Bilateral temporal poles are well-known core 
neural substrates for the formation of semantic representations (Lambon Ralph, Pobric, \& Jefferies, 2008) and the activation in right temporal pole in Hindi versus English, could possibly suggest a stronger anterior temporal lobe involvement in native versus the second language. On the other hand, the activation of the right caudate body in Hindi versus English contrast is interesting and is possibly an indication of visuo-spatial skill in reading Hindi as compared to English. Earlier work by Poldrack, Desmond, Glover, and Gabrielei (1998) has demonstrated that, the activation of the right caudate nucleus is a result of visuo-spatial skill learning. In their fMRI expriment on English mirror reading, unskilled participants were provided no training prior to the initial/first experiment. However, between the first and the second session, a long training was given. Poldrack et al. (1998) found increased activation in the right caudate nucleus between the first and the second session, and suggested a role for the right caudate nucleus in visuo-spatial skill learning. Such a role for the right caudate nucleus was also seen in a study on Japanese mirror reading in Kana wherein the subjects were provided training prior to the mirror-reading scanning sessions (Dong et al., 2000). The activation in the right caudate nucleus for Hindi as compared to English by late Hindi-English bilinguals is probably a demonstration of their visuo-spatial skills in reading Devanagari as compared to English. Additionally we also found shorter articulation times in Hindi. This was despite the fact reading in Hindi calls for greater visuospatial navigation and processing. We therefore attribute the shorter articulation times seen in Hindi to greater visuo-spatial skill demonstrated by the late bilinguals in reading Hindi. However, the complex visuo-spatial organization of Devanagari calls for extensive spatial and temporal sequencing of alphasyllabic elements during reading. Additionally, for certain words in Devanagari (not in English), the vowel precedes the consonant in writing but follows it in speech (for example, खिल, 'khil' meaning 'to blossom'). To deal with this disparity between spatial and temporal sequencing in Hindi, additional motor planning is required which we suspect is fulfilled by the left insula (BA 47/48, 9/12 subjects) (Dronkers, 1996).

Direct comparisons of Hindi and English also revealed activation in the superior temporal gyrus (STG) of the right hemisphere in 11/12 subjects. Hindi is an orthographically transparent language and the transparency of a writing system could influence the preference for either an assembled phonology strategy or an addressed phonology strategy in reading. Assembled phonology involves mapping of orthography to phonology prior to accessing lexical representation and lexical access is brought out through phonological mediation (Patterson \& Coltheart, 1987). Assembled phonology will be a default method favored by a transparent writing system such as Devanagari. In addressed phonology, the orthographic code directly activates the lexical representation, which is then followed by phonological access. English, which has an opaque orthography, favors the addressed phonology strategy. We expected high cortical activation in left superior temporal gyrus (LSTG) for Hindi along the lines of results reported by Meschyan and Hernandez (2006). We did not find such activation in the present study. There could be two probable explanations for this absence of activation in LSTG in Devanagari reading. All the participants in our study were highly proficient in Hindi and therefore it is possible that fluent readers even in a transparent orthography do not employ the 
assembled phonology strategy. The non-linear organization of Devanagari akshara may also constrain the phonological processing or phonological mediation strategy at the phonemic level. The second possibility is that the participants in the present study, who were not so fluent in English, were using the assembled phonology for reading in English and hence no contrast was found between transparent and opaque orthographies.

Though we find activation in STG, it is in the right hemisphere. A review of the imaging literature, suggests a role for right STG during rhythmic implementation of syllables (Riecker, Wildgruber, Dogil, Grodd, \& Ackermann, 2002). Since Hindi has been classified as a syllable-timed language (Bhatia, 1996; Das, Singh, \& Singh, 2008) we attribute the activation of STG in the right hemisphere to the syllable timed rhythm arising from the covert articulatory movements of Hindi. In contrast, relative to reading in Hindi, no orthography specific activation was seen for reading in English. One possibility could be that the reading network established for handling the complex spatial patterns of Devanagari overlaps considerably with that required for processing the linear alphabetic script of English. Our finding that processing in Hindi requires participation of visuo-spatial processing areas from the right hemisphere (BA 38 and caudate nucles) is in line with orthography specific activations seen for other scripts. For example, reading in Chinese, which uses a spatially complex, logographic script, showed participation of right occipital and fusiform areas (Liu et al., 2007). Liu and Perfetti (2003) have suggested that the spatial processing required to handle the complex visual forms of Chinese might be fulfilled by the right hemisphere. Similarly, Korean characters and words, which are non-linear and spatially complex show right hemisphere involvement (BA 8), which has been proposed to be responsible for processing of visuospatial (surface form) information of Korean words (Yoon, Cho, Chung, \& Park, 2005). Right hemisphere involvement for the processing of Japanese scripts Kanji (medial fusiform gyrus, BA 37) and Kana (occipital) have also been observed by Nakamura, Dehaene, Jobert, Bihan and Kouider (2005). We therefore reaffirm that, in order to deal with the spatially complex and highly non-linear characters of Devanagari, additional processing of visuo-spatial areas from the right hemisphere is elicited.

Additionally, the absence of speech-motor activation in the direct comparison of Hindi and English is also of interest. When compared with the results of Meschyan and Hernandez (2006), the absence of activation in speech-motor regions while reading a transparent orthography (in this case Hindi), provides support to their assertion that speech-motor activation is associated with articulatory motor effort and is a result of poor reading fluency.

In summary, reading fluency and orthography were found to influence reading phrases in Hindi and English. This paper presents the first report on the cortical activation patterns underlying the reading of Devanagari, an ancient writing system used by about 200 million people in South Asia. As seen for other visually complex orthographies like Chinese and Korean, reading in Devanagari also seems to recruit additional neural resources in the right hemisphere to cope with increased visuospatial processing. Clearly, more studies in alphasyllabary would help us in validating findings from other orthographic milieu and also in taking us closer to arriving at universal theory of reading. 
Acknowledgments We acknowledge Gurpreet Singh and S. Raghunathan for their support in image acquisition and Chetan Nagaraja for assistance in image analysis. We also wish to thank Ria de Blesser and Isabell Wartenburger, for their valuable comments on an earlier draft. The authors would also like to thank two anonymous referees whose comments greatly improved this manuscript. This research was supported by intramural funding from the National Brain Research Centre, India.

\section{References}

Bavelier, D., Corina, D., Jezzard, P., Padmanabhan, S., Clark, V. P., Karni, A., et al. (1997). Sentence reading: A functional MRI study at 4 tesla. Journal of Cognitive Neuroscience, 9, 664-686.

Bhatia, T. K. (1996). Colloquial Hindi. The complete course for beginners. London: Taylor and Francis.

Bolger, D. J., Perfetti, C. A., \& Schneider, W. (2005). Cross-cultural effect on the brain revisited: Universal structures plus writing system variation. Human Brain Mapping, 25, 92-104.

Booth, J. R., Burman, D. D., Meyer, J. R., Gitelman, D. R., Parrish, T. B., \& Mesulam, M. M. (2004). Development of brain mechanisms for processing orthographic and phonologic representations. Journal of Cognitive Neuroscience, 16, 1234-1249.

Callan, D. E., Tsytsarev, V., Hanakawa, T., Callan, A. M., Katsuhara, M., Fukuyama, H., et al. (2006). Song and speech: Brain regions involved with perception and covert production. Neuroimage, 31 , 1327-1342.

Chee, M. W. L., Hon, N., Lee, H.-L., \& Soon, C.-S. (2001). Relative language proficiency modulates BOLD signal change when bilinguals perform semantic judgements. Neuroimage, 13, 1155-1163.

Clarke, M. A. (1980). The short circuit hypothesis of ESL reading or when language competence interferes with reading performance. Journal of Modern Languages, 64, 203-209.

Das, T., Singh, L., \& Singh, N. C. (2008). Rhythmic structures of Hindi and English: New insights from a computational analysis. Progress in Brain Research, 168, 207-214.

Dietz, N. A., Jones, K. M., Gareau, L., Zeffiro, T., \& Eden, G. (2005). Phonological processing involves left posterior fusiform cortex. Human Brain Mapping, 26, 81-93.

Dong, Y., Fukuyama, H., Honda, M., Okada, T., Hanakawa, T., \& Nakamura, K. (2000). Essential role of the right superior parietal cortex in Japanese Kana mirror reading: An fMRI study. Brain, 123, 790-799.

Dronkers, N. F. (1996). A new brain region for coordinating speech articulation. Nature, 384, 159-161.

Economic Division, Ministry of Finance, Government of India. (2004). UNDP Human Development Report. New Delhi.

Fiez, J. A. (2000). Sound and meaning: How native language affects reading strategies. Nature Neuroscience, 3, 3-5.

Fiez, J. A., Balota, D. A., Raichle, M. E., \& Petersen, S. E. (1999). Effects of lexicality, frequency and spelling-to-sound consistency on the functional anatomy of reading. Neuron, 24, 205-218.

Frenck-Mestre, C., Anton, J. L., Roth, M., Vaid, J., \& Viallet, F. (2005). Articulation in early and late bilinguals' two languages: Evidence from functional magnetic resonance imaging. NeuroReport, 16, 761-765.

Friston, K. J., Frith, C. D., Frackowiak, R. S., \& Turner, R. (1995a). Characterizing dynamic brain responses with fMRI: A multivariate approach. Neuroimage, 2, 166-172.

Friston, K. J., Holmes, A. P., Worsley, K. J., Poline, J.-B., Frith, C. D., \& Frackowiak, R. S. J. (1995b). Statistical parametric maps in functional imaging: A general linear approach. Human Brain Mapping, 4, 189-210.

Goodman, K. S. (1973). Psycholinguistic universals in the reading process. In F. Smith (Ed.), Psycholinguistics and reading (pp. 21-29). New York: Holt, Rinehart and Winston.

Gottardo, A., Yan, B., Siegel, L. S., \& Wade, W. L. (2001). Factors related to English reading performance in children with Chinese as a first language: More evidence of a cross-language transfer of phonological processing. Journal of Educational Psychology, 93, 530-542.

Gracco, V. L., Tremblay, P., \& Pike, B. (2005). Imaging speech production using fMRI. Neuroimage, 26, 294-301.

Haller, S., Radue, E. W., Erb, M., Grodd, W., \& Kircher, T. (2005). Overt sentence production in eventrelated fMRI. Neuropsychologia, 43, 807-814.

Holm, A., \& Dodd, B. (1996). The effect of first written language on the acquisition of English literacy. Cognition, 59, 119-147. 
Kim, K. H., Relkin, N. R., Lee, K. M., \& Hirsch, J. (1997). Distinct cortical areas associated with native and second languages. Nature, 388, 171-174.

Klein, D., Zatorre, R. J., Milner, B., Meyer, E., \& Evans, A. C. (1995). Left putaminal activation when speaking a second language: Evidence from PET. NeuroReport, 5, 2295-2297.

Koda, K. (1994). Second language reading research: Problems and possibilities. Applied Psycholinguistics, $15,1-28$.

Lambon Ralph, M. A., Pobric, G., \& Jefferies, E. (2008). Conceptual knowledge is underpinned by the temporal pole bilaterally: Novel data from rTMS. Cerebral Cortex, 19, 832-838.

Liu, Y., Dunlap, S., Fiez, J., \& Perfetti, C. (2007). Evidence for neural accomodation to a writing system following learning. Human Brain Mapping, 28, 1223-1234.

Liu, Y., \& Perfetti, C. A. (2003). The time course of brain activity in reading English and Chinese: An ERP study of Chinese bilinguals. Human Brain Mapping, 18, 167-175.

MacKenzie, S. I., \& Tanaka-Ishii, K. (2007). Text entry systems: Mobility, accessibility, universality. New York: Academic Press.

Meschyan, G., \& Hernandez, A. E. (2006). Impact of language proficiency and orthographic transparency on bilingual word reading: An fMRI investigation. Neuroimage, 29, 1135-1140.

Nakamura, K., Dehaene, S., Jobert, A., Bihan, D. L., \& Kouider, S. (2005). Subliminal convergence of kanji and kana words: Further evidence for functional parcellation of the posterior temporal cortex in visual word perception. Journal of Cognitive Neuroscience, 17, 954-968.

Nelson, J., Liu, Y., Fiez, J., \& Perfetti, C. A. (2005). Learning to read Chinese as a second language recruits Chinese-specific visual word-form areas. Paper presented at the Society for the Scientific Study of Reading Conference, Toronto.

Neville, H. J., Bavelier, D., Corina, D., Rauschecker, J., Karni, A., Lalwani, A., et al. (1998). Cerebral organization for language in deaf and hearing subjects: Biological constraints and effects of experience. Proceedings of National Academy of Sciences USA, 95, 922-929.

Oldfield, R. C. (1971). The assessment and analysis of handedness: The Edinburgh inventory. Neuropsychologia, 9, 97-113.

Padakannaya, P., \& Joshi, M. (1995). Orthography and reading in Kannada: A Dravidian language. In I. Taylor \& D. Olson (Eds.), Scripts and reading: Reading and learning to read world's scripts (pp. 95-108). Boston: Kluwer Academic Publishers.

Padakannaya, P., \& Mohanty, A. K. (2004). Indian orthography and teaching how to read: A Psycholinguistic framework. Psychological Studies, 49, 262-271.

Padakannaya, P., Rekha, D., Nigam, R., \& Karanth, P. (1993). Phonological awareness, orthography and literacy. In R. Scholes (Ed.), Literacy: Linguistic and cognitive perspectives (pp. 55-70). Hillslade, NJ: Erlbaum Associates.

Patel, P. G. (2004). Exploring reading acquisition and dyslexia in India. New Delhi: Sage.

Patterson, K. E., \& Coltheart, M. (1987). Phonological processes in reading. A tutorial review. In M. Coltheart (Ed.), Attention and performance XII: The psychology of reading (pp. 171-200). Hillsdale, NJ: Erlbaum Associates.

Paulesu, E., McCrory, E., Fazio, F., Menoncello, L., Brunswick, N., Cappa, S. F., et al. (2000). A cultural effect on brain function. Nature Neuroscience, 3, 91-96.

Penny, W. D., Holmes, A. P., \& Friston, K. J. (2003). Random effects analysis. In R. S. J. Frackowiak, K. J. Friston, C. D. Frith, R. Dolan, C. J. Price, S. Zeki, J. Ashburner, \& W. D. Penny (Eds.), Human brain function (pp. 843-850). New York: Academic Press.

Perani, D., Paulesu, E., Galles, N. S., Dupoux, E., Dehaene, S., \& Bettinardi, V. (1998). The bilingual brain. Proficiency and age of acquisition of the second language. Brain, 121, 1841-1852.

Perfetti, C. A. (2003). The universal grammar of reading. Scientific Studies of Reading, 8, 3-24.

Perfetti, C. A., Liu, Y., Fiez, J., Nelson, J., \& Bolger, D. J. (2007). Reading in two writing systems: Accomodation and assimilation of the brain's reading network. Bilingualism: Language and Cognition, 10, 131-146.

Perfetti, C. A., Zhang, S., \& Berent, I. (1992). Reading in English and Chinese: Evidence for a "universal" phonological principle. In R. Frost \& L. Katz (Eds.), Orthography, phonology, morphology and meaning (pp. 227-248). Amsterdam, Netherlands: North Holland.

Poeppel, D. (1996). A critical review of PET studies of phonological processing. Brain and Language, 55, 317-351.

Poldrack, R. A., Desmond, J. A., Glover, G. H., \& Gabrielei, J. D. (1998). The neural basis of visual skill learning: an fMRI study of mirror reading. Cerebral Cortex, 8, 1-10. 
Ravizza, S. M., Delgado, M. R., Chein, J. M., Becker, I. T., \& Fiez, J. A. (2004). Functional dissociaitons within the inferior parietal cortex in verbal working memory. Human Brain Mapping, 22, 562-573.

Riecker, A., Wildgruber, D., Dogil, G., Grodd, W., \& Ackermann, H. (2002). Hemispheric lateralization effects of rhythm implementation during syllable repetitions: An fMRI study. Neuroimage, 16, $169-176$.

Sheela, N. (2007). Segmental awareness and learning to read conjunct consonants in Kannada. M.Phil. Dissertation, Department of Psychology, University of Mysore, Mysore, India.

Singh, P., Solanki, R., \& Bhatnagar, P. (2008). BIS-11A- Hindi version: A preliminary study of impulsivity in rural and urban Indian adolescents. Indian Journal of Psychiatry, 50, 96-99.

Smith, E. E., \& Jonides, J. (1999). Storage and executive processes in the frontal lobes. Science, 282, $1657-1661$.

Sproat, R., \& Padakannaya, P. (2008). Script indices. In S. Narayanan, A. K. Gupta, \& J. Pandey (Eds.), Advances in cognitive science (pp. 62-70). New Delhi: Sage.

Talairach, J., \& Tournoux, P. (1988). Co-planar stereotaxic atlas of the human brain. New York: Thieme.

Tan, L. H., Liard, A. R., Li, K., \& Fox, P. T. (2005). Neuroanatomical correlates of phonological processing of Chinese characters and alphabetic words: A meta-analysis. Human Brain Mapping, $25,83-91$.

Tan, L. H., Spinks, J. A., Feng, C.-M., Siok, W. T., Perfetti, C. A., Xiong, J., et al. (2003). Neural systems of second language reading are shaped by native language. Human Brain Mapping, 18, 158-166.

Vaid, J. (2002). Bilingualism. In V. S. Ramachandra (Ed.), Encyclopedia of the human brain (Vol. 1, pp. 417-434). San Diego: Elsevier.

Vaid, J., \& Gupta, A. (2002). Exploring word recognition in a semi-alphabetic script: The case of Devanagari. Brain and Language, 81, 679-690.

Vaid, J., \& Hull, R. (2002). Re-envisioning the bilingual brain using functional neuroimaging: Methodological and interpretive issues. In F. Fabbro (Ed.), Advances in the neurolinguistics of bilingualism: A festschrift for Michel Paradis (pp. 315-355). Udine, Italy: Udine University Press.

Wang, M., \& Geva, E. (2003). Spelling performances of Chinese ESL children: Lexical and visual orthographic processes. Applied Psycholinguistics, 24, 1-25.

Weinreich, U. (1953). Languages in contact: findings and problems. London: Mouton.

Yoon, H. W., Cho, K., Chung, J., \& Park, H. W. (2005). Neural mechanisms of Korean word reading: A functional magnetic resonance imaging study. Neuroscience Letters, 373, 206-211. 